\title{
Inverse transient thermoelastic deformations in thin circular plates
}

\author{
A K TIKHE ${ }^{1}$ and $\mathrm{K}$ C DESHMUKH ${ }^{2}$ \\ ${ }^{1}$ Department of Mathematics, Late KZS Science College, Bramhani 441501 \\ (Kalmeshwar), India \\ ${ }^{2}$ Department of Mathematics, Post Graduate Department of Teaching, Nagpur \\ University, Nagpur 440 010, India \\ e-mail: kcdeshmukh2000@rediffmail.com
}

MS received 10 March 2005; revised 10 August 2005

\begin{abstract}
This paper deals with the determination of unknown heating temperatures and temperature distributions on the upper surface of a thin circular plate, defined as $0 \leq r \leq a,-b / 2 \leq z \leq b / 2$. The expressions of unknown heating temperatures and temperature distributions are obtained in series form, involving Bessel's functions with the help of the integral transform technique. Thermoelastic deformations are discussed with the help of temperature and are illustrated numerically.
\end{abstract}

Keywords. Inverse transient; thermoelastic deformation

\section{Introduction}

The inverse thermoelastic problem consists of determination of the temperature, the heat flux on the boundary surfaces of the solid when the conditions of the displacement and stresses are known at some points of the solid under consideration. Grysa \& Cialkowski (1980), Grysa \& Kozlowski (1982) investigated one-dimensional transient thermoelastic problems and derived the heating temperature and heat flux on the surface of an isotropic infinite slab. The problems of normal deflection of an axisymmetrically heated circular plate in the case of fixed and simply supported edges have been considered by Boley \& Weiner (1960). Further, Roychoudhuri (1973) has succeeded in determining the normal deflection of a thin clamped circular plate due to ramp-type heating of a concentric circular region of the upper face. Ishihara \& Noda (1997) has considered the theoretical analysis of thermoelastoplastic deformation of a circular plate due to partially distributed heat supply. In this paper, we modify the work of Roychoudhari (1973) and discuss the thermoelastic deformation on the upper surface in a thin circular plate subjected to heating. The results, obtained in series form involving Bessel's functions, are illustrated numerically. 


\section{Analysis}

\subsection{Inverse transient heat conduction problem}

Consider a thin circular plate of radius $a$ and thickness $b$ defined as $0 \leq r \leq a,-b / 2 \leq z \leq$ $b / 2$. The plate is initially at zero temperature.

The temperature $T(r, z, t)$ of the plate at time $t$, satisfying the heat conduction equation is as

$$
\frac{\partial^{2} T}{\partial r^{2}}+\frac{1}{r} \frac{\partial T}{\partial r}+\frac{\partial^{2} T}{\partial z^{2}}=\frac{1}{k} \frac{\partial T}{\partial t},
$$

with the initial condition, boundary conditions and interior condition respectively, as

$$
\begin{aligned}
t & =0, \quad T(r, z, t)=0, \\
r & =a, \quad\left[(\partial T / \partial r)+h_{e} T\right]=0, \\
z & =-b / 2, \quad\left[(\partial T / \partial z)-h_{s_{1}} T\right]=g(r, t) \text { (unknown), } \\
z & =b / 2, \quad\left[(\partial T / \partial z)+h_{s_{2}} T\right]=0, \\
\text { and } \quad z & =\xi, T(r, z, t)=f(r, t) \text { (known). }
\end{aligned}
$$

where $-(b / 2)<\xi<(b / 2), k$ is thermal diffusivity of the material of the plate and $h_{s_{1}}, h_{s_{2}}$ and $h_{e}$ are the relative heat transfer coefficients of the plate on the upper surface $(z=-b / 2)$, lower surface $(z=b / 2)$ and outer curved surface $(r=a)$ respectively.

Equations (1) to (6) constitute the mathematical formulation of the inverse heat conduction problem.

\subsection{Thermoelastic problem}

In this subsection, we formulate the thermoelastic behaviour of a circular plate. We assume that the plate in sufficiently thin. Therefore, we can introduce the assumption that the plane perpendicular to the neutral plane $(z=0)$ before deformation remains the plane perpendicular to it after deformation, and that the axial stress $\sigma_{z z}$ is negligible compared to the other stress components. Furthermore $\sigma_{r \theta}, \sigma_{\theta z}, \epsilon_{r \theta}$ and $\epsilon_{\theta z}$ are all zero because the plate deforms axisymmetrically, where $\epsilon_{r \theta}=\left(\sigma_{r \theta} / 2 G\right)$ and $\epsilon_{\theta z}=\left(\sigma_{\theta z} / 2 G\right)$, and $G$ is the shear modulus.

The differential equation satisfied by force function $F$ is defined (Ishiharar Noda 1997) as

$$
\frac{\mathrm{d}}{\mathrm{d} r}\left(\nabla^{2} F\right)=-\frac{\mathrm{d}}{\mathrm{d} r} N_{T},
$$

where

$$
\nabla^{2}=\left(\frac{\mathrm{d}^{2}}{\mathrm{~d} r^{2}}+\frac{1}{r} \frac{\mathrm{d}}{\mathrm{d} r}\right),
$$

and the thermal resultant force, $N_{T}$, is defined as

$$
N_{T}=\int_{-b / 2}^{b / 2} \alpha E T \mathrm{~d} z,
$$


where $\alpha$ and $E$ are the coefficient of linear thermal expansion and Young's modulus respectively.

The boundary condition concerning the in-plane deformation is given by

$$
r=a, \quad \mathrm{~d} F / \mathrm{d} r=0, \quad F=0 .
$$

The differential equation satisfied by the deflection $\omega(r, t)$ is

$$
D \frac{\mathrm{d}}{\mathrm{d} r}\left(\nabla^{2} \omega\right)=-\frac{1}{(1-v)} \frac{\mathrm{d}}{\mathrm{d} r} M_{T},
$$

where $D$ is the flexural rigidity of the plate denoted by

$$
D=\frac{E b^{3}}{12\left(1-v^{2}\right)}
$$

and the resultant thermal moment $M_{T}$ is defined as

$$
M_{T}=\int_{-b / 2}^{b / 2} E \alpha T z \mathrm{~d} z
$$

The boundary conditions concerning the out-of-plane deformation are

$$
\text { and } \left.\quad \begin{array}{rl}
r & =a, M_{r}=0 \\
r & =0, \omega=0
\end{array}\right\}
$$

where

$$
M_{r}=D\left(\frac{\mathrm{d}^{2} \omega}{\mathrm{d} r^{2}}+\frac{v}{r} \frac{\mathrm{d} \omega}{\mathrm{d} r}\right)+\frac{M_{T}}{1-v}=0 .
$$

Equations (7) to (13) constitute the mathematical formulation of the thermoelastic problem.

\section{Solution of the inverse heat conduction problem}

3.1 Determination of the temperature function, $T(r, z, t)$ and unknown heating temperature, $g(r, t)$

On applying the finite Hankel transform and Laplace transform to (1) to (6) and then obtaining the inverses of the resultant equations, we obtain the expressions for temperature distribution and unknown heating temperature respectively, as

$$
\begin{aligned}
T(r, z, t)= & \sum_{n=1}^{\infty} \sum_{m=1}^{\infty} A_{m n}\left(\xi_{n}, \lambda_{m}\right) \cdot \phi_{m}(n, t) \cdot J_{0}\left(\xi_{n} r\right) \\
& \times\left[-\lambda_{m} \cos \left(\frac{\lambda_{m}}{\xi-(b / 2)} \cdot(z-(b / 2))\right)\right. \\
& \left.+h_{s_{1}}\left(\xi-\frac{b}{2}\right) \sin \left(\frac{\lambda_{m}}{\xi-(b / 2)}(z-(b / 2))\right)\right]
\end{aligned}
$$


and

$$
g(r, t)=\sum_{n=1}^{\infty} \sum_{m=1}^{\infty} B_{m n}\left(\xi_{n}, \lambda_{m}\right) \cdot \phi_{m}(n, t) \cdot J_{0}\left(\xi_{n} r\right)
$$

where

$$
\begin{aligned}
A_{m n}\left(\xi_{n}, \lambda_{m}\right)= & \frac{-4 K}{(\xi-(b / 2))^{2}}\left[\frac{\lambda_{m}}{a^{2}\left\{\left[J_{0}\left(\xi_{n} a\right)\right]^{2}+\left[J_{1}\left(\xi_{n} a\right)\right]^{2}\right\}}\right] \\
& \times\left[\frac{1}{\lambda_{m} \sin \left(\lambda_{m}\right)+\left[h_{s_{2}}(\xi-(b / 2))-1\right] \cos \left(\lambda_{m}\right)}\right], \\
B_{m n}\left(\xi_{n}, \lambda_{m}\right)= & \frac{-4 K}{(\xi-(b / 2))^{3}}\left[\frac{\lambda_{m}}{a^{2}\left\{\left[J_{0}\left(\xi_{n} a\right)\right]^{2}+\left[J_{1}\left(\xi_{n} a\right)\right]^{2}\right\}}\right] \\
& \times \frac{\left[-\lambda_{m}^{2}+h_{s_{1}} h_{s_{2}}(\xi-(b / 2))^{2}\right] \sin \left(\lambda_{m} b /[\xi-(b / 2)]\right)}{\lambda_{m} \sin \left(\lambda_{m}\right)+\left[h_{s_{2}}(\xi-(b / 2))-1\right] \cos \left(\lambda_{m}\right)}
\end{aligned}
$$

and

$$
\phi_{m}(n, t)=\int_{0}^{t} \bar{f}\left(\xi_{n}, \tau\right) \cdot \exp \left\{-K\left(\xi_{n}^{2}+\frac{\lambda_{m}^{2}}{(\xi-(b / 2))^{2}}\right)(t-\tau)\right\} d \tau,
$$

where $m, n$ are positive integers, $\xi_{n}$ is the $n$th positive root of the transcendental equation

$$
-\xi J_{1}(\xi a)+h_{e} J_{0}(\xi a)=0,
$$

and $\lambda_{m}$ is the $m$ th positive root of the transcendental equation

$$
\lambda \cos (\lambda)-h_{s_{2}}(\xi-(b / 2)) \sin (\lambda)=0 .
$$

\section{Solution of the thermoelastic problem}

\subsection{Determination of force component, $F(r, t)$}

Substituting the value of temperature $T(r, z, t)$ from (14) in (8), we obtain

$$
\begin{aligned}
N_{T}= & -\alpha E\left(\xi-\frac{b}{2}\right)^{2} \sum_{m=1}^{\infty} \sum_{n=1}^{\infty}\left[\frac{A_{m n}\left(\xi_{n}, \lambda_{m}\right)}{\lambda_{m}} \phi_{m}(n, t)\right. \\
& \times\left\{\left(\frac{\lambda_{m}}{(\xi-(b / 2))}\right) \sin \left(\frac{\lambda_{m} b}{\xi-(b / 2)}\right)\right. \\
& \left.\left.+h_{s_{1}}\left[1-\cos \left(\frac{\lambda_{m} b}{\xi-(b / 2)}\right)\right]\right\} J_{0}\left(\xi_{n} r\right)\right],
\end{aligned}
$$

where $A_{m n}\left(\xi_{n}, \lambda_{m}\right)$ and $\phi_{m}(n, t)$ are as in (16) and (18) respectively. 
On substituting (19) in (7) and using (9), we obtain force component $F(r, t)$ as

$$
\begin{aligned}
F= & \alpha E\left(\xi-\frac{b}{2}\right)^{2} \sum_{m=1}^{\infty} \sum_{n=1}^{\infty}\left[\frac{A_{m n}\left(\xi_{n}, \lambda_{m}\right)}{\lambda_{m} \xi_{n}} \phi_{m}(n, t)\right. \\
& \left.\times\left\{\left(\frac{\lambda_{m}}{\xi-(b / 2)}\right) \sin \left(\frac{\lambda_{m} b}{\xi-(b / 2)}\right)+h_{s_{1}}\left[1-\cos \left(\frac{\lambda_{m} b}{\xi-(b / 2)}\right)\right]\right\}\right] \\
& \times\left[J_{0}\left(\xi_{n} r\right)-J_{0}\left(\xi_{n} a\right)+\frac{\xi_{n}}{2 a}\left(r^{2}-a^{2}\right) J_{1}\left(\xi_{n} a\right)\right] .
\end{aligned}
$$

4.2 Determination of deflection function, $\omega(r, t)$

On substituting the value of temperature $T(r, z, t)$ from (14) in (12), we get

$$
\begin{aligned}
M_{T}= & \alpha E \sum_{m=1}^{\infty} \sum_{n=1}^{\infty}\left[A _ { m n } ( \xi _ { n } , \lambda _ { m } ) \phi _ { m } ( n , t ) \left\{\frac{b}{2 \lambda_{m}}\left(\xi-\frac{b}{2}\right)\right.\right. \\
& \times\left[\left(\frac{\lambda_{m}}{\xi-(b / 2)}\right) \sin \left(\frac{\lambda_{m} b}{\xi-(b / 2)}\right)-h_{s_{1}}\left[1+\cos \left(\frac{\lambda_{m} b}{\xi-(b / 2)}\right)\right]\right] \\
& +\frac{\left(\xi-\frac{b}{2}\right)^{2}}{\lambda_{m}^{2}}\left[\lambda_{m}\left(\cos \left(\frac{\lambda_{m} b}{\xi-b / 2}\right)-1\right)\right. \\
& \left.\left.\left.+\left(\xi-\frac{b}{2}\right) h_{s_{1}} \sin \left(\frac{\lambda_{m} b}{\xi-b / 2}\right)\right]\right\} J_{0}\left(\xi_{n} r\right)\right] .
\end{aligned}
$$

Finally, we substitute the value of $M_{T}$ from (21) in (10) and use (13), and get deflection function $\omega(r, t)$ as

$$
\begin{aligned}
\omega= & \frac{\alpha E}{D(1-v)} \sum_{m=1}^{\infty} \sum_{n=1}^{\infty}\left[\frac { A _ { m n } ( \xi _ { n } , \lambda _ { m } ) } { \xi _ { n } } \phi _ { m } ( n , t ) \left\{\frac{b}{2 \lambda_{m}}\left(\xi-\frac{b}{2}\right)^{2}\right.\right. \\
& \times\left[\left(\frac{\lambda_{m}}{\xi-(b / 2)}\right) \sin \left(\frac{\lambda_{m} b}{\xi-(b / 2)}\right)-h_{s_{1}}\left[1+\cos \left(\frac{\lambda_{m} b}{\xi-(b / 2)}\right)\right]\right. \\
& +\frac{\left(\xi-\frac{b}{2}\right)^{2}}{\lambda_{m}^{2}}\left[\lambda_{m}\left(\cos \left(\frac{\lambda_{m} b}{\xi-b / 2}\right)-1\right)\right. \\
& \left.\left.\left.+\left(\xi-\frac{b}{2}\right) h_{s_{1}} \sin \left(\frac{\lambda_{m} b}{\xi-b / 2}\right)\right]\right\}\right] \\
& \times\left[J_{0}\left(\xi_{n} r\right)-\frac{r^{2} \xi_{n}}{2(1+v)}\left\{\left(1-\xi_{n}\right) J_{0}\left(\xi_{n} a\right)+\frac{(1-v)}{a} J_{1}\left(\xi_{n} a\right)\right\}-1\right],
\end{aligned}
$$

and the angle of variation with time as

$$
\theta=-\mathrm{d} \omega / \mathrm{d} r,
$$


i.e.

$$
\begin{aligned}
\theta= & \frac{\alpha E}{D a\left(1-v^{2}\right)} \sum_{m=1}^{\infty} \sum_{n=1}^{\infty}\left[A _ { m n } ( \xi _ { n } , \lambda _ { m } ) \phi _ { m } ( n , t ) \left\{\frac{b}{2 \lambda_{m}}\left(\xi-\frac{b}{2}\right)^{2}\right.\right. \\
& \times\left[\left(\frac{\lambda_{m}}{\xi-(b / 2)}\right) \sin \left(\frac{\lambda_{m} b}{\xi-(b / 2)}\right)-h_{s_{1}}\left[1+\cos \left(\frac{\lambda_{m} b}{\xi-(b / 2)}\right)\right]\right. \\
& +\frac{\left(\xi-\frac{b}{2}\right)^{2}}{\lambda_{m}^{2}}\left[\lambda_{m}\left(\cos \left(\frac{\lambda_{m} b}{\xi-b / 2}\right)-1\right)\right. \\
& \left.\left.\left.+\left(\xi-\frac{b}{2}\right) h_{s_{1}} \sin \left(\frac{\lambda_{m} b}{\xi-b / 2}\right)\right]\right\}\right] \\
& \times\left[a(1+v) J_{1}\left(\xi_{n} r\right)+r\left\{a\left(1-\xi_{n}\right) J_{0}\left(\xi_{n} a\right)+(1-v) J_{1}\left(\xi_{n} a\right)\right\}\right] .
\end{aligned}
$$

\section{Special case}

Let

$$
f(r, t)=\left(1-e^{-A t}\right)\left(r^{2}-a^{2}\right)^{2},
$$

where $A>0$ is constant.

We apply the finite Hankel transform defined as (Sneddon 1972) to get

$$
\bar{f}\left(\xi_{n}, \tau\right)=\frac{a^{2}}{\xi_{n}^{2}}\left[\left(\frac{64}{a \xi_{n}^{3}}-\frac{8 a}{\xi_{n}}\right) J_{1}\left(\xi_{n} a\right)-\frac{32}{\xi_{n}^{2}} J_{0}\left(\xi_{n} a\right)\right]\left(1-e^{-A \tau}\right) .
$$

On using (25) in (18), we obtain

$$
\begin{aligned}
\phi_{m}(n, t)= & \frac{a^{2}}{\xi_{n}^{2}}\left[\left(\frac{64}{a \xi_{n}^{3}}-\frac{8 a}{\xi_{n}}\right) J_{1}\left(\xi_{n} a\right)-\frac{32}{\xi_{n}^{2}} J_{0}\left(\xi_{n} a\right)\right] \\
& \times\left[\frac{1-e^{-k\left(\xi_{n}^{2}+\frac{\lambda_{m}^{2}}{(\xi-(b / 2))^{2}}\right) t}}{k\left(\xi_{n}^{2}+\frac{\lambda_{m}^{2}}{(\xi-(b / 2))^{2}}\right)}+\frac{e^{-A t}-e^{-k\left(\xi_{n}^{2}+\frac{\lambda_{m}^{2}}{(\xi-(b / 2))^{2}}\right) t}}{A-k\left(\xi_{n}^{2}+\frac{\lambda_{m}^{2}}{(\xi-(b / 2))^{2}}\right)}\right]
\end{aligned}
$$

Finally, substituting (26), (16), (17) in (14), (15), (20), (22) and (23), we obtain the expressions for temperature distribution, unknown heating temperature, force component, thermal deflection and angle of variation respectively, as

$$
\begin{aligned}
& T(r, z, t) \\
& \quad=\frac{-4 K}{(\xi-(b / 2))^{2}} \sum_{m=1}^{\infty} \sum_{n=1}^{\infty}\left[\frac{\lambda_{m}\left[\left(\left(64 / a \xi_{n}^{3}\right)-\left(8 a / \xi_{n}\right)\right) J_{1}\left(\xi_{n} a\right)-\left(32 / \xi_{n}^{2}\right) J_{0}\left(\xi_{n} a\right)\right]}{\left.\xi_{n}^{2}\left\{\left[J_{0}\left(\xi_{n} a\right)\right]^{2}+\left[J_{1} \xi_{n} a\right)\right]^{2}\right\}}\right] J_{0}\left(\xi_{n} r\right)
\end{aligned}
$$




$$
\begin{aligned}
& \times \frac{-\lambda_{m} \cos \left[\frac{\lambda_{m}}{(\xi-(b / 2))}\left(z-\frac{b}{2}\right)\right]+h_{s_{2}}\left(\xi-\frac{b}{2}\right) \sin \left[\frac{\lambda_{m}}{(\xi-(b / 2))}\left(z-\frac{b}{2}\right)\right]}{\lambda_{m} \sin \left(\lambda_{m}\right)+\left[h_{s_{2}}(\xi-(b / 2))-1\right] \cos \left(\lambda_{m}\right)} \\
& \times \frac{1-e^{-k\left(\xi_{n}^{2}+\frac{\lambda_{m}^{2}}{(\xi-(b / 2))^{2}}\right) t}}{k\left[\xi_{n}^{2}+\frac{\lambda_{m}^{2}}{(\xi-(b / 2))^{2}}\right]}+\frac{e^{-A t}-e^{-k\left(\xi_{n}^{2}+\frac{\lambda_{m}^{2}}{(\xi-(b / 2))^{2}}\right) t}}{A-K\left(\xi_{n}^{2}+\frac{\lambda_{m}^{2}}{(\xi-(b / 2))^{2}}\right)},
\end{aligned}
$$

$g(r, t)$

$$
\begin{aligned}
& =\frac{-4 K}{(\xi-(b / 2))^{3}} \sum_{m=1}^{\infty} \sum_{n=1}^{\infty}\left[\frac{\lambda_{m}\left[\left(\left(64 / a \xi_{n}^{3}\right)-\left(8 a / \xi_{n}\right)\right) J_{1}\left(\xi_{n} a\right)-\left(32 / \xi_{n}^{2}\right) J_{0}\left(\xi_{n} a\right)\right]}{\left.\xi_{n}^{2}\left\{\left[J_{0}\left(\xi_{n} a\right)\right]^{2}+\left[J_{1} \xi_{n} a\right)\right]^{2}\right\}}\right] J_{0}\left(\xi_{n} r\right) \\
& {\left[-\lambda_{m}^{2}+h_{s_{1}} h_{s_{2}}(\xi-(b / 2))^{2}\right] \sin \left(\lambda_{m} b /(\xi-b / 2)\right)} \\
& \times \frac{+\lambda_{m}(\xi-(b / 2))\left(h_{s_{1}}+h_{s_{2}}\right) \cos \left(\lambda_{m} b /(\xi-b / 2)\right)}{\lambda_{m} \sin \left(\lambda_{m}\right)+\left[h_{s_{2}}(\xi-(b / 2))-1\right] \cos \left(\lambda_{m}\right)} \\
& \times \frac{1-\exp \left\{-k\left[\xi_{n}^{2}+\frac{\lambda_{m}^{2}}{(\xi-(b / 2))^{2}}\right] t\right\}}{k\left[\xi_{n}^{2}+\frac{\lambda_{m}^{2}}{(\xi-(b / 2))^{2}}\right]}+\frac{\exp (-A t)-\exp \left\{-k\left(\xi_{n}^{2}+\frac{\lambda_{m}^{2}}{(\xi-(b / 2))^{2}}\right) t\right\}}{A-K\left(\xi_{n}^{2}+\frac{\lambda_{m}^{2}}{(\xi-(b / 2))^{2}}\right)} \\
& F=-4 k \alpha E \sum_{m=1}^{\infty} \sum_{n=1}^{\infty}\left[\frac{\left[\left(\left(64 / a \xi_{n}^{3}\right)-\left(8 a / \xi_{n}\right)\right) J_{1}\left(\xi_{n} a\right)-\left(32 / \xi_{n}^{2}\right) J_{0}\left(\xi_{n} a\right)\right]}{\left.\xi_{n}^{3}\left\{\left[J_{0}\left(\xi_{n} a\right)\right]^{2}+\left[J_{1} \xi_{n} a\right)\right]^{2}\right\}}\right] \\
& \times \frac{\left[\lambda_{m} /(\xi-b / 2)\right] \sin \left(\lambda_{m} b /(\xi-b / 2)\right)+h_{s_{1}}\left[1-\cos \left(\lambda_{m} b /(\xi-b / 2)\right)\right]}{\lambda_{m} \sin \left(\lambda_{m}\right)+\left[h_{s_{2}}(\xi-(b / 2))-1\right] \cos \left(\lambda_{m}\right)} \\
& \times\left[\left\{J_{0}\left(\xi_{n} r\right)-J_{0}\left(\xi_{n} a\right)\right\}+\frac{\xi_{n}}{2 a}\left(r^{2}-a^{2}\right) J_{1}\left(\xi_{n} a\right)\right] \\
& \times \frac{1-e^{-k\left(\xi_{n}^{2}+\frac{\lambda_{m}^{2}}{(\xi-(b / 2))^{2}}\right) t}}{k\left[\xi_{n}^{2}+\frac{\lambda_{m}^{2}}{(\xi-(b / 2))^{2}}\right]}+\frac{e^{-A t}-e^{-k\left(\xi_{n}^{2}+\frac{\lambda_{m}^{2}}{(\xi-(b / 2))^{2}}\right) t}}{A-K\left(\xi_{n}^{2}+\frac{\lambda_{m}^{2}}{(\xi-(b / 2))^{2}}\right)}
\end{aligned}
$$

$\omega(r, t)$

$$
\begin{aligned}
= & \frac{-4 K \alpha E}{D(1-v)(\xi-(b / 2))^{2}} \sum_{m=1}^{\infty} \sum_{n=1}^{\infty}\left\{\frac{\lambda_{m}}{\xi_{n}^{3}\left\{\left[J_{0}\left(\xi_{n} a\right)\right]^{2}+\left[J_{1}\left(\xi_{n} a\right)\right]^{2}\right\}}\right\} \\
& {\left[\frac{b}{2 \lambda_{m}}(\xi-b / 2)^{2}\left\{\frac{\lambda_{m}}{(\xi-(b / 2))} \sin \left(\frac{\lambda_{m} b}{\xi-(b / 2)}\right)-h_{s_{1}}\left[1+\cos \left(\frac{\lambda_{m} b}{\xi-(b / 2)}\right)\right]\right\}\right.} \\
& \times \frac{+\frac{(\xi-(b / 2))^{2}}{\lambda_{m}^{2}}\left[\lambda_{m}\left(\cos \left(\frac{\lambda_{m} b}{(\xi-(b / 2))}-1\right)+h_{s_{1}}(\xi-b / 2) \sin \frac{\lambda_{m} b}{(\xi-(b / 2))}\right]\right]}{\lambda_{m} \sin \left(\lambda_{m}\right)+\left[h_{s_{2}}(\xi-(b / 2))-1\right] \cos \left(\lambda_{m}\right)}
\end{aligned}
$$




$$
\begin{aligned}
& \times\left[\left(\frac{64}{a \xi_{n}^{3}}-\frac{8 a}{\xi_{n}}\right) J_{1}\left(\xi_{n} a\right)-\frac{32}{\xi_{n}^{2}} J_{0}\left(\xi_{n} a\right)\right] \\
& {\left[J_{0}\left(\xi_{n} r\right)-\frac{r^{2} \xi_{n}}{2(1+v)}\left\{\left(1-\xi_{n}\right) J_{0}\left(\xi_{n} a\right)+\frac{(1-v)}{a} J_{1}\left(\xi_{n} a\right)\right\}-1\right]} \\
& \times \frac{1-e^{-k\left(\xi_{n}^{2}+\frac{\lambda_{m}^{2}}{(\xi-(b / 2))^{2}}\right) t}}{k\left[\xi_{n}^{2}+\frac{\lambda_{m}^{2}}{(\xi-(b / 2))^{2}}\right]}+\frac{e^{-A t}-e^{-k\left(\xi_{n}^{2}+\frac{\lambda_{m}^{2}}{(\xi-(b / 2))^{2}}\right) t}}{A-K\left(\xi_{n}^{2}+\frac{\lambda_{m}^{2}}{(\xi-(b / 2))^{2}}\right)}
\end{aligned}
$$

and

$$
\begin{aligned}
\theta= & \frac{-4 k \alpha E}{D a\left(1-v^{2}\right)(\xi-(b / 2))^{2}} \\
& \times \sum_{m=1}^{\infty} \sum_{n=1}^{\infty}\left[\frac{\lambda_{m}}{\xi_{n}^{2}} \frac{\left[\left(\left(64 / a \xi_{n}^{3}\right)-\left(8 a / \xi_{n}\right)\right) J_{1}\left(\xi_{n} a\right)-\left(32 / \xi_{n}^{2}\right) J_{0}\left(\xi_{n} a\right)\right]}{\left.\left\{\left[J_{0}\left(\xi_{n} a\right)\right]^{2}+\left[J_{1} \xi_{n} a\right)\right]^{2}\right\}}\right] \\
& \quad\left[\frac{b}{2 \lambda_{m}}(\xi-b / 2)^{2}\left\{\frac{\lambda_{m}}{(\xi-(b / 2))} \sin \left(\frac{\lambda_{m} b}{\xi-(b / 2)}\right)-h_{s_{1}}\left[1+\cos \left(\frac{\lambda_{m} b}{\xi-(b / 2)}\right)\right]\right\}\right. \\
& \times \frac{+\frac{(\xi-(b / 2))^{2}}{\lambda_{m}^{2}}\left[\lambda_{m}\left(\cos \left(\frac{\lambda_{m} b}{(\xi-b / 2)}-1\right)+h_{s_{1}}(\xi-b / 2) \sin \frac{\lambda_{m} b}{(\xi-b / 2)}\right]\right]}{\lambda_{m} \sin \left(\lambda_{m}\right)+\left[h_{s_{2}}(\xi-(b / 2))-1\right] \cos \left(\lambda_{m}\right)} \\
& \times \frac{\left.1-e^{-k\left(\xi_{n}^{2}+\frac{\lambda_{m}^{2}}{(\xi-(b / 2))^{2}}\right) t}+\frac{e^{-A t}-e^{-k\left(\xi_{n}^{2}+\frac{\lambda_{m}^{2}}{(\xi-(b / 2))^{2}}\right) t}}{k\left[\xi_{n}^{2}+\frac{\lambda_{m}^{2}}{(\xi-(b / 2))^{2}}\right]}+r\left\{a\left(1-\xi_{n}\right) J_{0}\left(\xi_{n} a\right)+(1-v) J_{1}\left(\xi_{n} a\right)\right\}\right]}{A-K\left(\xi_{n}^{2}+\frac{\lambda_{m}^{2}}{(\xi-(b / 2))^{2}}\right)},
\end{aligned}
$$

\section{Numerical results and discussions}

Numerical calculations have been carried out for a steel plate (SN 50C) for which the material constants are as follows:

$$
\begin{aligned}
& a=1 \mathrm{~m}, b=0.2 \mathrm{~m}, A=0 \cdot 3, \xi=0.05 \mathrm{~m}, \pi=3 \cdot 14, K=15.9 \times 10^{-6}\left(\mathrm{~m}^{2} \mathrm{~s}^{-1}\right), \\
& h_{s_{1}}=1, h_{s_{2}}=0, h_{e}=1, \alpha=11.6 \times 10^{-6}\left(\mathrm{~K}^{-1}\right), E=215(\mathrm{GPa}), v=0 \cdot 281,
\end{aligned}
$$

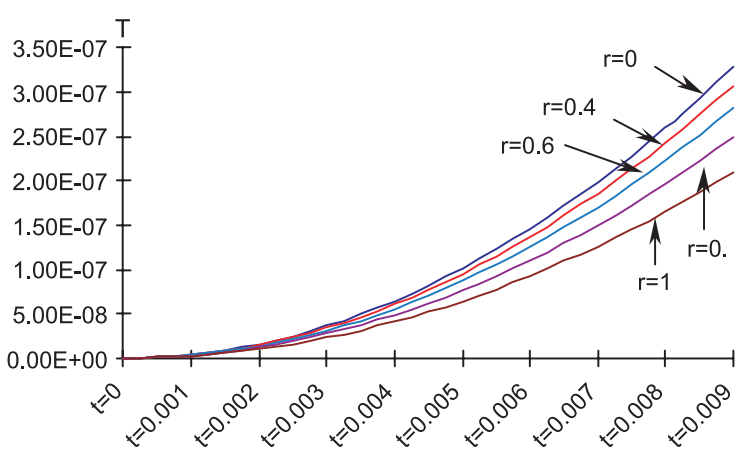

Figure 1. Variation of temperature $T$ versus time $t$. 


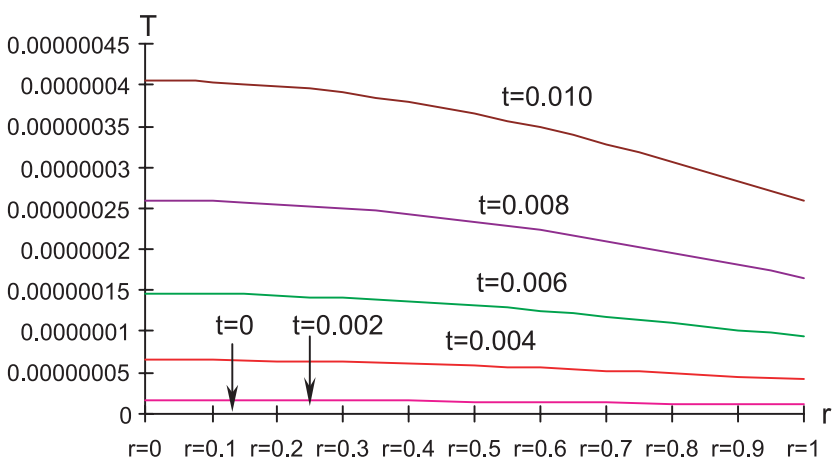

Figure 2. Variation of temperature $T$ versus radius $r$.

and time $t$ is in seconds. The plate is thin due to it being of one-fifth thickness of the largest dimension in the work of Nowinski (1978). The first five roots of the transcendental equation

$$
-\xi J_{1}(\xi a)+h_{e} J_{0}(\xi a)=0
$$

(Özisik 1968, p. 491) are $\xi_{1}=1 \cdot 2558, \xi_{2}=4 \cdot 0795, \xi_{3}=7 \cdot 1558, \xi_{4}=10 \cdot 2710, \xi_{5}=$ 13.3984 and the first five roots of the transcendental equation

$$
-\lambda \cos \lambda+h_{s_{2}}(\xi-b / 2) \sin \lambda=0
$$

i.e. $\lambda \cot \lambda=-c$, where $c=0$ (constant) (Özisik 1968, p. 482) are $\lambda_{1}=1.5708, \lambda_{2}=$ $4.7124, \lambda_{3}=7.8540, \lambda_{4}=10.9956, \lambda_{5}=14.1372$. Using the numerical values of the above material constants and the roots of the transcendental equations, the temperature distribution, the thermal deflection and the angle of variation are evaluated. Due to the temperature effect on the upper surface, the fluctuations in deflection and angle of variation are shown. The variations are shown in figures 1 to 4 .

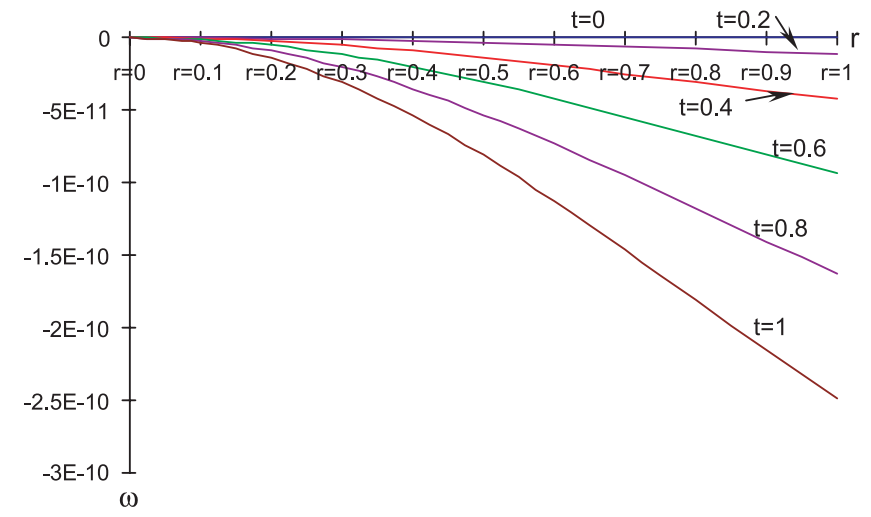

Figure 3. Variation of deflection $\omega$ versus radius $r$. 


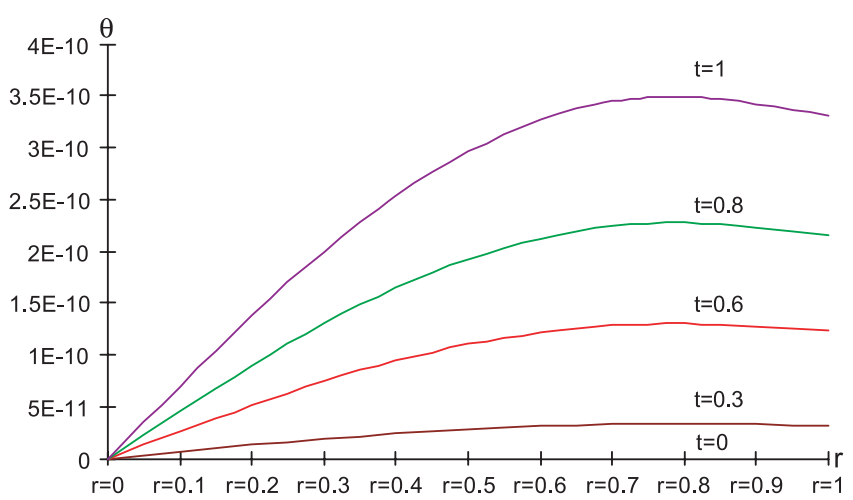

Figure 4. Angle of variation $\theta$ versus radius $r$.

\section{Concluding remarks}

In this work we have dealt with the determination of force component, thermal deflection and angle of variation of a thin circular plate on the upper surface due to heating. The expressions for the temperature distribution, unknown heating temperature, force component, thermal deflection and angle of variation are obtained in series form involving Bessel's functions. An integral transform technique is employed to solve this inverse problem. The numerical results are prescribed graphically for the temperature distribution, the thermal deflection and the angle of variation. This inverse problem may be useful in engineering applications particularly for industrial machines subjected to heating such as the main shaft of a lathe, turbines and rolling mills. Any particular case of special interest can be derived by assigning suitable values to the parameters and functions in the expressions (14), (15), (20), (22) and (23).

We thank the anonymous referees for the suggestions to improve the paper. We thank Dr P C Wankhede for his valuable guidance in the work. We acknowledge the University Grants Commission, New Delhi for partial assistance under a research project scheme.

\section{References}

Boley B A, Weiner J H 1960 Theory of thermal stresses (New York: John Wiley and Sons)

Deshmukh K C, Wankhede P C 1998 An inverse transient problem of a quasi-static thermal deflection of a thin clamped circular plate. Bull. Pure Appl. Sci. 17: 1, 165-172

Grysa K W, Cialkowski M J 1980 On a certain inverse problem of temperature and thermal stress field. Acta Mech. 36: 169-185

Grysa K W, Kozlowski Z 1982 One dimensional problems of temperature and heat flux determination at the surfaces of a thermoelastic slab, Part 1: The analytical solutions. Nucl. Engrg. Des. 74: 1-14

Ishihara M, Noda N 1997 Theoretical analysis of thermoelastoplastic deformation of a circular plate due to a partially distributed heat supply. J. Thermal Stresses 20: 203-225

Khobragade N L, Deshmukh K C 2003 An inverse quasi-static thermal deflection problem for a thin clamped circular plate. J. Thermal Stresses (accepted) 
Nowinski J L 1978 Theory of thermoelasticity with application (Alphen aan den Rijn: Sijthof Noordhoff) p. 407

Özisik M N 1968 Boundary value problem of the heat conduction (Scranton, PA: Int. Textbook Co.) Roychoudhuri S K 1973 A note on the quasi-static thermal deflection of a thin clamped circular plate due to ramp-type of heating of a concentric circular region of the upper face. J. Franklin Inst. 296: 3: 213-219

Sneddon I N 1972 The use of integral transforms (New York: McGraw-Hill) 